\title{
Sound-Intensity-Dependent Compensation for the Small Interaural Time Difference Cue for Sound Source Localization
}

\author{
Eri Nishino, ${ }^{1}$ Rei Yamada, ${ }^{1}$ Hiroshi Kuba, ${ }^{1}$ Hiroyuki Hioki, ${ }^{2}$ Takahiro Furuta, ${ }^{2}$ Takeshi Kaneko, ${ }^{2}$ and Harunori Ohmori ${ }^{1}$ \\ Departments of ${ }^{1}$ Physiology and ${ }^{2}$ Morphological Brain Science, Faculty of Medicine, Kyoto University, Kyoto 606-8501, Japan
}

Interaural time difference (ITD) is a major cue for sound source localization. However, animals with small heads experience small ITDs, making ITD detection difficult, particularly for low-frequency sound. Here, we describe a sound-intensity-dependent mechanism for compensating for the small ITD cues in the coincidence detector neurons in the nucleus laminaris (NL) of the chicken aged from 3 to $29 \mathrm{~d}$ after hatching. The hypothesized compensation mechanisms were confirmed by simulation. In vivo single-unit recordings revealed an improved contrast of ITD tuning in low-best-frequency $(<1 \mathrm{kHz})$ NL neurons by suppressing the firing activity at the worst ITD, whereas the firing rate was increased with increasing sound intensity at the best ITD. In contrast, level-dependent suppression was so weak in the middle- and high-best-frequency ( $\geq 1 \mathrm{kHz}$ ) NL neurons that loud sounds led to increases in firing rate at both the best and the worst ITDs. The suppression of firing activity at the worst ITD in the low-best-frequency neurons required the activation of the superior olivary nucleus ( $\mathrm{SON}$ ) and was eliminated by electrolytic lesions of the SON. The frequency-dependent suppression reflected the dense projection from the SON to the low-frequency region of NL. Thus, the small ITD cues available in low-frequency sounds were partly compensated for by a sound-intensity-dependent inhibition from the SON.

Key words: sound localization; interaural time difference; nucleus laminaris; nucleus magnocellularis; coincidence detection; superior olivary nucleus

\section{Introduction}

Detecting the interaural difference cues in time [interaural time difference (ITD)] and intensity is critical for sound source localization. These cues are the function of the distance between the left and right ears of an animal and are generally very small, especially in animals having small heads. The largest ITD occurs when the sound source faces one ear, and is less than several hundred microseconds in most species (Klump, 2000; Konishi, 2003; Joris and Yin, 2007).

ITD is first processed in the nucleus laminaris (NL) in birds and in the nucleus medial superior olive (MSO) in mammals (Konishi, 2003). Neurons in these nuclei act as coincidence detectors, and their firing rates change as a function of ITD, being maximal when spikes from each ear arrive simultaneously (the best ITD) and minimal at the worst ITD. The firing rate at the worst ITD was sometimes lower than that for the monaural stimulation or even for the spontaneous activity (Goldberg and Brown, 1969; Yin and Chan, 1990). Some contribution of inhibitory systems has been suggested to improve the ITD detection

\footnotetext{
Received Sept. 26, 2007; revised May 28, 2008; accepted June 6, 2008.

This work was supported by Ministry of Education, Science, and Technology Grants-in-Aid 17023027 (H.0.) 17021021 and 17700368 (H.K.), and 18700381 (R.Y.). We thank Prof. Catherine E. Carr and Prof. Christine Köppl for reading this manuscript.

Correspondence should be addressed to Harunori Ohmori at the above address. E-mail: ohmori@nbiol.med.kyoto-u.ac.jp.

D0I:10.1523/JNEUROSCI.4398-07.2008

Copyright $\odot 2008$ Society for Neuroscience $\quad 0270-6474 / 08 / 287153-12 \$ 15.00 / 0$
}

(Grothe, 2000; Konishi, 2003). In gerbils, precisely timed glycinergic inhibitory inputs relayed by the medial nucleus of the trapezoid body (MNTB) were reported to be pivotal in ITD processing for low-frequency sound (Brand et al., 2002). In birds, the fast inhibitory neurons like the principal cells of MNTB do not exist in the ITD processing circuit, whereas around the perikaryon and proximal dendrites of NL are concentrated GABA terminals (Carr et al., 1989; Code et al., 1989). The major source of these terminals is the superior olivary nucleus (SON), which receives the input proportional to the sound intensity from the nucleus angularis (NA), and makes an inhibitory innervation to NL (Lachica et al., 1994; Yang et al., 1999; Burger et al., 2005). Moreover, GABA is reported to facilitate coincidence detection from the findings in in vitro experiments in NL (Hyson et al., 1995; Funabiki et al., 1998). By simulation, the inhibition from SON is proposed to increase the threshold of neural activity in NL of the barn owl to make the ITD sensitivity tolerant to the sound intensity (Peña et al., 1996). It is suggested further that SON activity may balance the effects of interaural intensity difference in processing ITD (Burger et al., 2005; Dasika et al., 2005). Although various compensatory functions of SON activity have been proposed so far, it is still not clear how these functions actually shape the ITD tuning of NL neurons in a living animal. In this study, we made single-unit recordings from NL of the chicken across frequencies and examined the ITD sensitivity in the presence or in the absence of SON activity. We found that the ITD coding was improved by the inhibition from SON in a sound-intensity- 
dependent manner, and with some assistance of simulation, we propose a neural mechanism of how the small ITD cues are compensated for by the sound intensity cues.

\section{Materials and Methods}

Preparation. Chickens of 3-7 d (juvenile) and 23-29 d (young adult) after hatching were anesthetized with an intramuscular injection of chloral hydrate $(160 \mathrm{mg} / \mathrm{kg})$ and were used for experiments. Surgical procedures were the same as those previously described (Fukui et al., 2006) and conformed to the guiding principles for the care and use of animals in the field of physiological sciences set by the Japanese Physiological Society.

ITD-tuning curves were recorded from 38 control units (29 from juvenile chickens and 7 from young adult chickens) and 21 units after SON lesion from juvenile chickens. Most analyses were made on stable units, from which ITD-tuning curves were measured at multiple sound intensities; these comprised 21 control units ( 14 from juvenile and 7 from young adult chickens) and 13 units from juvenile chickens after SON lesions.

Sound stimulation. Acoustic stimulus was made as previously described (Fukui et al., 2006). Briefly, a pair of small earphones (EF-1935; Knowles Electronics) was attached to the hollow ear bars and used for acoustic stimulation, and the sound intensity was measured by a pair of small microphones (BT-1751; Knowles Electronics) equipped with a probe tube to the ear bar. The sound pressure level (SPL) was calibrated using a standard microphone (type 4939; Bruel \& Kjaer). The stimuli were either pure-tone or white-noise sound of $80 \mathrm{~ms}$ duration, with $5 \mathrm{~ms}$ rise and fall of a sine wave-shaped envelope. White noise stimulus was applied to detect neurophonics while approaching NL. Binaural stimulation of the same SPL was applied, at sound intensities varying from 8 to $108 \mathrm{~dB}$ SPL by $10-20 \mathrm{~dB}$ steps. The ITD was varied in reference to the contralateral sound, in steps of one-sixth of the period of pure-tone stimuli. Sound stimulation and data acquisition were controlled by customized software written in MATLAB.

Electrophysiology. All experiments were performed in an electrically shielded soundproof box, placed within a soundproof room. The background noise level within the soundproof box evaluated by a standard microphone (type 4190; Bruel \& Kjaer) was practically flat, and the power was $1.7 \pm 4.0 \mathrm{~dB}$ SPL (mean $\pm \mathrm{SD}$ ) at frequencies from $100 \mathrm{~Hz}$ to $1 \mathrm{kHz}$.

A technique of loose patch recording was adopted (Peña et al., 1996). Patch pipettes were made from quartz glass capillaries (100-70-10; Sutter Instrument) and filled with an artificial CSF (concentrations in mM: 138 $\mathrm{NaCl}, 2.5 \mathrm{KCl}, 2.5 \mathrm{CaCl}_{2}, 1 \mathrm{MgCl}_{2}, 10 \mathrm{HEPES}, 26$ glucose); the range of resistance was 2-8 M $\Omega$, and the impedance of the electrode was continuously monitored. Electrodes were advanced vertically with a motordriven micromanipulator (PC-5N; Narishige). A small positive pressure was applied to the patch pipette while approaching NL. When a small action potential was detected, the positive pressure was released and the electrode was advanced carefully in $2 \mu \mathrm{m}$ steps until the unit size was maximized. A small negative pressure was maintained during recording. Neuronal signals were recorded with Axoclamp-2B amplifier (Molecular Devices) and bandpass filtered between $150 \mathrm{~Hz}$ and $10 \mathrm{kHz}$ (two-pole). The data were sampled at $50 \mathrm{kHz}$ with 12-bit resolution.

Extracting unit activities of NL. NL was located $\sim 200-300 \mu \mathrm{m}$ ventral to the nucleus magnocellularis (NM), and both units responded to the sound stimulus. However, NM unit responses were large, excited only ipsilaterally, and were not sensitive to ITD. Recording sites were occasionally confirmed by ejecting a fluorescent dye ionophoretically with a sharp electrode or by electrolysis.

Best frequency, rate threshold, and vector strength. After isolating a unit, the "best frequency" (BF) of the unit was determined by presenting a pure-tone sound stimulus at $200 \mathrm{~Hz}$ intervals. BF was measured as the sound frequency that generated the highest firing rate in the unit. This made each BF truncated at $200 \mathrm{~Hz}$, unless otherwise described. Firing threshold ("rate threshold") was defined as the sound intensity to induce firing rate at the best ITD higher than the spontaneous firing level by 40 spikes/s (see Fig. 6A) (Warchol and Dallos, 1990; Fukui et al., 2006). "Vector strength" (VS) was calculated as a measure of the phase-locked firing of units (see Fig. 6B) (Goldberg and Brown, 1969), from spikes occurring between 10 and $80 \mathrm{~ms}$ of the sound stimulus, provided that the phase locking was statistically significant (Rayleigh test; $p<0.05$ ).

Classification of NL units. Based on the tonotopic observations, we previously classified NL into three BF regions (Rubel and Parks, 1975; Kuba et al., 2005): low BF ( $<1 \mathrm{kHz})$, middle BF (1-2.5 kHz), and high BF $(>2.5 \mathrm{kHz})$. However, we could not find a clear distinction between the middle-BF and the high-BF units in this study as for the peak-trough contrast of ITD-tuning curves (see Fig. 2C), the effects of SON lesion (see Fig. $5 J$ ), and the ITD sensitivity (see Fig. $7 B$ ). The density of vesicular GABA transporter (VGAT)-positive particles per cell apparently graded along the frequency regions (see Fig. 3I); however, the density was distinct in the low-BF region. The area of SON projection and the number of VGAT-positive particles per region of interest in the low-BF region were also distinguishable (see Fig. $3 G$ ). We therefore categorized NL units in two classes, as low $\mathrm{BF}<1 \mathrm{kHz}$, and middle-high $\mathrm{BF} \geq 1 \mathrm{kHz}$, in this study.

Responsiveness to ITD, peristimulus time histogram, and period histogram. The ITD at the level of tympanic membrane is known to be different from that applied acoustically, and was likely enhanced by the interference through the interaural canal in the bird, particularly for the lowfrequency sound (Hyson et al., 1994). However, in this study, we evaluated the "responsiveness to ITD" as the dynamic range of firing responses within $\pm 100 \mu$ s ITD that was applied acoustically (see Fig. 7 A, shaded area), after normalization of the firing rate by the maximum firing rate of each unit. This is because we have not measured the effective ITD at the level of tympanic membrane, and the acoustically applied ITD was the only exact ITD we could define. ITD of $\pm 100 \mu$ s was close to the physiologically relevant ITD that was determined physically by the head size of the chicken. "Peristimulus time histogram" (PSTH) (bin width, 1 ms) and "period histogram" (bin width, $1 / 10$ of a period) of unit activities were measured at each ITD. The ITD and the phase of period histogram were calculated in reference to the contralateral sound.

Evaluation of suppression of the unit activity during sound stimulation. "Suppression" of unit activity at the best and the worst ITDs was measured as the ratio of spike number in the first $25 \mathrm{~ms}$ of sound responses to that in the last $25 \mathrm{~ms}$, and was the strongest when the ratio was 0 and was weaker toward 1 (see Fig. 5I). For units from which ITD-tuning curve was measured at multiple SPLs, the suppression at the maximum SPL was measured.

Lesion and red fluorescent protein labeling of SON. SON was located $0.7-1 \mathrm{~mm}$ lateral and $2-3 \mathrm{~mm}$ ventral from the high-frequency region of $\mathrm{NL}$, and the depth was $\sim 8-9 \mathrm{~mm}$ from the surface of the cerebellum (supplemental Fig. 2, available at www.jneurosci.org as supplemental material). We first detected the high-frequency region of NL, and then approached SON by monitoring the field potential responses to sound stimuli while advancing a tungsten electrode. The electrode was made by inserting a heavy-polyimide-coated tungsten wire ( $15 \mu \mathrm{m}$ diameter; California Fine Wire) into a microelectrode made from a glass capillary of $1.5 \mathrm{~mm}$ outer diameter (GD-1.5; Narishige); the resistance was $\sim 1 \mathrm{M} \Omega$, and the wire tip protruded $\sim 10 \mu \mathrm{m}$ from the capillary tip of $20-30 \mu \mathrm{m}$ diameter At the location where the largest auditory response was recorded, a lesion or labeling of SON was made. Because SON neurons are known to project mainly to ipsilateral NL, NA, and NM (Monsivais et al., 2000; Burger et al., 2005), we lesioned SON ipsilateral to NL recording sites by passing a constant current of $50 \mu \mathrm{A}$ for $100 \mathrm{~s}$. We began unit recordings $1 \mathrm{~h}$ later. In SON labeling, the glass pipette was filled with PBS containing recombinant Sindbis virus expressing a fluorescent protein mRFP1 (monomeric red fluorescent protein) (courtesy of Dr. R. Y. Tsien, University of California, San Diego, La Jolla, CA) with a membrane-targeted palmitoylation signal of GAP43 (Furuta et al., 2001); the virus was designed by and the cDNA was provided by H. Hioki, T. Furuta, and T. Kaneko. A positive pressure was applied to eject the Sindbis virus-mRFP (RFP) solution until the field potential decreased to $20 \%$, and then two pulses of $\pm 5 \mu \mathrm{A}$ of $4 \mathrm{~s}$ duration and $1 \mathrm{~s}$ interval were applied, and the electrode was left still for 15-20 min. The animal was allowed to survive for the next $12 \mathrm{~h}$ to $2 \mathrm{~d}$.

Fixation. After electrophysiological experiments, the animal was fixed by perfusing transcardially with $4 \%$ formaldehyde in PBS. For immunohistochemistry of vesicular GABA transporter (VGAT11-A; Alpha Diag- 
nostic), the fixation was made using a modified solution: $2 \%(\mathrm{w} / \mathrm{v})$ formaldehyde, $0.01 \%(\mathrm{w} / \mathrm{v})$ glutaraldehyde in PBS. After fixation, 50- to 100 $\mu \mathrm{m}$-thick brain slice preparations were made on the following day for general histological examination. For immunohistochemistry, brain slices of 20-30 $\mu \mathrm{m}$ thicknesses were made by cryomicrotome (Leica CM $3050)$ after cryoprotection with $30 \%(\mathrm{w} / \mathrm{w})$ sucrose in PBS.

VGAT immunostaining. Slices were incubated overnight with 1:1000 diluted anti-VGAT antibody in PBS containing $0.3 \%(\mathrm{v} / \mathrm{v})$ Triton X-100, $0.5 \%(\mathrm{w} / \mathrm{v})$ carrageenan, and $0.5 \%(\mathrm{v} / \mathrm{v})$ donkey serum (PBS-XCD), and then for $2 \mathrm{~h}$ with $10 \mu \mathrm{g} / \mathrm{ml}$ Alexa 488-conjugated anti-rabbit-IgG in PBS-XCD. Slices were mounted onto glass slides, coverslipped, and observed under a confocal laser-scanning microscope (LSM5 PASCAL; Carl Zeiss) with optical slice thicknesses of $<1.0 \mu \mathrm{m}$. Z-sectioning images were acquired at $1.0 \mu \mathrm{m}$ intervals.

Counting VGAT particles. Using VGAT-immunostained slices, a series of two images were stacked and the number of VGAT-positive particles was counted automatically from images of $2.0 \mu \mathrm{m}$ thicknesses for the region of interest $(43.6 \times 53.4 \mu \mathrm{m})$, using Image-J. After setting a threshold by eye, particles were counted in the range of $10-100$ pixels ( 1 pixel $=$ $0.051 \mu \mathrm{m}^{2}$ ). The number of neurons within each region of interest was determined by eye. Neurons were immunostained as described by Kuba et al. (2005) by antibody to Kv1.2 (anti-Kv1.2 channel rabbit polyclonal antibody; Alamone Labs; APC-010).

VGAT and RFP overlay. To analyze the overlapping of RFP-positive particles and VGAT, the slices from SON-labeled animals by virus injection were stained by anti-VGAT antibody, and single planes $(1.0 \mu \mathrm{m})$ of confocal images were analyzed with off-line software (LSM Image Browser; Carl Zeiss). Both RFP-positive particles and overlaid VGATpositive particles were counted manually.

Statistical analyses. Statistical evaluations were made using Student's $t$ test. Values are presented as mean \pm SEM. In figures, the numbers in parentheses are the numbers of data; ${ }^{* *}$ and ${ }^{\star}$ are the statistical significance of $p<0.01$ and $p<0.05$, respectively; and error bars are SEM.

Simulation. Simulation was made by NEURON 5.9 as previously reported (Kuba et al., 2006). Briefly, bilateral excitatory inputs of the same conductance from NM and the ipsilateral inhibitory input from SON were made on the soma in the control. Because the SON lesion was made ipsilateral to the recording site of NL, the SON lesion was expected to reduce the inhibitory inputs to the ipsilateral NL and NM (Monsivais et al., 2000; Lu and Trussell, 2001; Burger et al., 2005). The reduction of SON activity to NM might facilitate activation of NM neurons, and this was included in the simulation by increasing the size of ipsilateral EPSP.

Simulation was made presuming a neuron of $500 \mathrm{~Hz} \mathrm{BF}$, and synaptic inputs were made at $2 \mathrm{~ms}$ intervals. Inhibitory conductance was made proportional to the excitatory conductance and was included both as the steady-state conductance and as IPSPs, although the incorporation of IPSP was not essential for reproducing the ITD-tuning properties of low-BF units. The conductance for steady-state inhibition was 4.5 times and that for IPSP was 0.1 times the conductance of EPSP. IPSP was phase-independent to EPSPs, and one IPSP was generated at random timing in one stimulus cycle.

EPSP and IPSP followed an $\alpha$-function $\left[=g_{\text {syn }} \times t / \tau_{\text {syn }} \times \exp (1-\right.$ $\left.t / \tau_{\text {syn }}\right)$ ] with a time constant of EPSC decay of $0.29 \mathrm{~ms}$ and that of IPSC of $5 \mathrm{~ms}$. Reversal potential for the inhibitory input was $-55 \mathrm{mV}$, although a more positive $\mathrm{E}_{\mathrm{Cl}}{ }^{-}$has been reported in NM (Monsivais et al., 2000; Lu and Trussell, 2001). Firing activities were calculated by changing ITDs and were demonstrated as a period histogram, ITD-tuning curves, and the peak-trough contrast with inhibition and without inhibition (see Fig. 9). ITD was created by placing a delay to ipsilateral EPSP, following the definition of ITD in our experiments.

\section{Results}

\section{Sound intensity dependence of ITD tuning in NL}

When sound stimuli were applied binaurally, the NL unit activity was modulated periodically in a manner depending on ITD (Fig. 1). Figure $1 A$ shows the raster plots from a low-BF neuron $(\mathrm{BF}<$ $1 \mathrm{kHz}$ ) when a strong tonal stimulus of BF (98 dB SPL; $200 \mathrm{~Hz}$ ) was applied with ITD. The PSTH shows a robust firing activity at the best ITD, whereas the firings were suppressed completely after an onset response when a stimulus of worst ITD was applied (Fig. $1 B$ ). The suppression lasted for some time after the stimulation regardless of ITD (Fig. $1 \mathrm{~A}$ ). In contrast, in the middle- and high-BF neurons ( $\mathrm{BF} \geq 1 \mathrm{kHz}$ ), the unit activity was robust both at the best and the worst ITD and spontaneous activities remained after the stimulus (Fig. $1 F, G$ ) when a strong tonal stimulus of BF ( $68 \mathrm{~dB}$ SPL; $2400 \mathrm{~Hz}$ ) was applied. The best and the worst ITDs were determined from the ITD-tuning curves (Figs. $1 C, H)$.

The ITD-tuning curve was SPL dependent both in the low-BF and middle-high-BF units (Fig. $1 C, H$ ). In the low-BF units, the firing activity was increased at the best ITD with the SPL, whereas the firing rate at the worst ITD was reduced lower than the spontaneous firing level and the contrast of ITD tuning was large at the strong SPL (Fig. 1C). In contrast, in the middle-high-BF units, the firing rates were increased with SPL both at the best and the worst ITDs, and the contrast of ITD tuning disappeared at the strong SPL (Fig. $1 \mathrm{H})$. Figure 1, C $(200 \mathrm{~Hz}$; P5) and $H(2400 \mathrm{~Hz}$; $\mathrm{P} 4)$, are the ITD-tuning curves from the juvenile chickens. Figure $1, D$ and $I$, show ITD-tuning curves from the young adult chickens for low-BF unit (400 Hz; P23) and a middle-high-BF unit $(1800 \mathrm{~Hz} ; \mathrm{P} 27)$, respectively. These ITD-tuning curves were similarly dependent on SPL as in the juvenile chickens (Fig. 1C,H).

Figure $1 E$ plots the firing rate at the best (solid lines) and the worst ITD (broken lines) as a function of SPL from the five units demonstrated in Figure 1. The firing rates at the best ITDs increased with SPL both in the low- (blue solid lines) and the middle-high-BF (black and red solid lines) units. Firing rates at the worst ITD of the middle-high-BF unit (black broken lines) increased with SPL and approached the firing level of best ITD of the unit (Fig. $1 H$, open circles for juvenile; $I$, filled diamonds for a young adult chicken), with the exception of the one middlehigh-BF unit from a young adult chicken in which the firing rate and its contrast between the best and the worst ITD remained SPL independent (red lines). The ITD-tuning curve of this unit is presented in Figure $1 \mathrm{~J}$, and its property will be detailed in a later section. In contrast, in the low-BF units, the firing rate was decreased at the worst ITD when the SPL was increased (Fig. 1C, blue open squares for juvenile; $D$, blue filled triangles for a young adult chicken). We have tested SPL dependence of ITD tunings both in the juvenile chickens (P3-P7; $n=6$ from low-BF and 8 from middle-high-BF units) and in the young adult chickens (P23-P29; 3 from low-BF and 4 from middle-high-BF units). All the low-BF units regardless of ages of the animal and most of the middle-high-BF units (all eight juvenile units, and three of four young adult units) demonstrated a corresponding pattern of SPL dependence, and the contrast of ITD tuning was increased in the low-BF units, whereas it was decreased in the middle-high-BF units with SPL.

\section{Peak-trough contrast of ITD tunings}

The contrast of ITD tuning was measured as a function of SPL (Fig. 2). A peak-trough contrast was defined here as the difference of firing rates between the best ITD (peak) and the worst ITD (trough) from each ITD-tuning curve, and was normalized by the maximal firing rate of the unit after subtraction of the spontaneous firing rate. The spontaneous firing level was subtracted to mark the suppression at the worst ITD, because by the subtraction of the spontaneous firing rate, the peak-trough contrast was $>1$ when the firing rate of worst ITD was lower than the spontaneous level. These contrasts were plotted against the SPL relative to the rate threshold (Fig. $2 A, B$ ). In the low-BF units (Fig. 
$2 A)$, the peak-trough contrast increased with the sound level, and in most units the contrast became close to or larger than 1 (juvenile, circles connected by solid lines; young adult, diamonds and broken lines). However, in the middle-high-BF units (Fig. $2 B$ ), the peaktrough contrast behaved differently: In most units, the maximum contrast was achieved at the intermediate stimulus intensities, and the contrast was reduced to a lower level at the strong SPL. This pattern of SPL dependence was observed in all juvenile units, and in three of four young adult units (diamonds and broken lines).

One young adult unit of middle-high BF (BF, $2000 \mathrm{~Hz}$; P26) maintained the contrast of ITD tunings when the SPL was raised (Fig. $1 J$ ), and the firing rate both at the best and the worst ITD was practically SPL independent (Fig. $1 E$, red lines). Consequently, the unit exhibited a substantial level of peak-trough contrast even at the strong SPL ( $80 \mathrm{~dB}$ above the rate threshold) (Fig. $2 B$, red). The tolerance to sound intensity of this middle-high-BF unit appears similar to the one reported in the adult barn owl (Peña et al., 1996). This may reflect some maturational effects in the middlehigh-BF NL neurons (Lippe and Rubel, 1985). Nevertheless, the level of peak-trough contrast was far smaller than that observed in the low-BF units, and was 0.5 at most. This may reflect that the firing rate at the worst ITD was substantially higher than the spontaneous firing level when SPL was high. Therefore, the unit appears different from both the low-BF and the middle-high-BF units and seems to have an SPL dependence somewhat intermediate between the low- and the middle-high-BF units.

There was a difference in the SPL dependence of peak-trough contrast between the low-BF units and the middle-high-BF units (Fig. 2A,B), and the difference was apparently most prominent at the strong SPL. Then, we evaluated the peak-trough contrast at the maximum SPL of each unit (Fig. 2C). In most low-BF units, the contrast was close to or larger than 1; this indicated that the firing rate was suppressed close to or below the spontaneous level at the worst ITD. The contrast was decreased with the increase of $\mathrm{BF}(p<0.01$; $n=14$ from P3-P7 and 7 from P23-P29), and there was no difference between the juvenile and young adult chickens $(p=0.45$ for low$\mathrm{BF}$, and $p=0.38$ for middle-high-BF units). Figure $2 D$ shows the spontaneous firing level (filled columns), and the firing rate at the worst ITD after normalization by the spontaneous firing rate at the SPL of best peaktrough contrast (open columns). The spontaneous firing rate was not different between the juvenile and the young adult $(p=0.26$ for low $\mathrm{BF}$, and $p=0.25$ for middle-high $\mathrm{BF}$ ). Moreover, the spontaneous firing rate was not different between the low- and
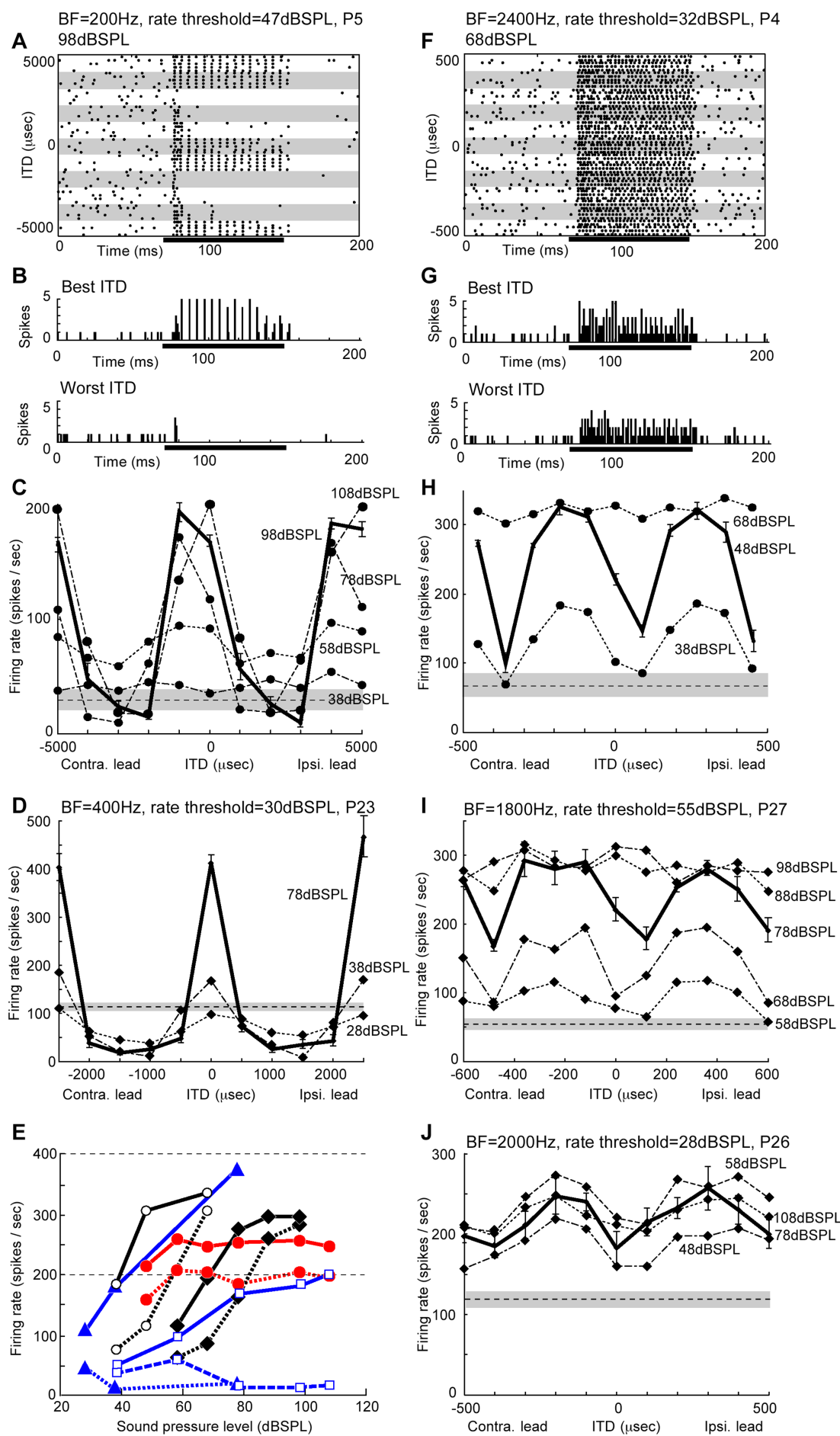

Figure 1. ITD tuning to a pure-tone sound stimulus of best frequency. $\boldsymbol{A}-\boldsymbol{D}, \mathrm{A}$ low-BF neuron $(\boldsymbol{A}-\boldsymbol{C}, 200 \mathrm{~Hz}$ from a juvenile chicken, $\mathrm{P} 5 ; \boldsymbol{D}, 400 \mathrm{~Hz}$ from a young adult chicken, $\mathrm{P} 23)$. $\boldsymbol{F}$ - $\boldsymbol{J}$, A middle-high-BF neuron ( $\boldsymbol{F}-\boldsymbol{H}, 2400 \mathrm{~Hz}$ from a juvenile chicken, P4; $I, 1800 \mathrm{~Hz}, \mathrm{P} 27 ; J, 2000 \mathrm{~Hz}, \mathrm{P} 26) . A, \boldsymbol{F}$, Raster plots. The gray and white bands indicate a set of five repeats of ITD. The horizontal bars indicate sound presentation here and the subsequent PSTHs. $\boldsymbol{B}, \mathbf{G}$, PSTHs at the best (top) and the worst ITDs (bottom) obtained at $98 \mathrm{dBSPL}(\boldsymbol{B})$ and $68 \mathrm{dBSPL}(\boldsymbol{G})$. Bin width, $1 \mathrm{~ms}$. C, $\boldsymbol{D}, \boldsymbol{H}, \boldsymbol{I}, \mathbf{J}$, ITD-tuning curves. ITD-tuning curve of best contrast is indicated by a solid line with error bars of SEM, and the horizontal broken line and the shade indicate the mean \pm SEM of spontaneous firings. Stimulus SPL and the rate threshold are indicated. $\boldsymbol{E}$, Firing rates of the best ITD (solid lines) and the worst ITD (broken lines) as a function ofSPL, from five units presented in this figure. The symbols identify units; the open symbols are units from juvenile chickens, and the filled symbols are units from young adult chickens. Contra., Contralateral; Ipsi., ipsilateral.
middle-high-BF units $(p=0.26)$, whereas the firing rate was suppressed at the worst ITD and became lower than the spontaneous firing level in most low-BF units $(0.82 \pm 0.29 ; n=9 ; p<$ 0.01 ). This strong suppression in low-BF units might indicate the 

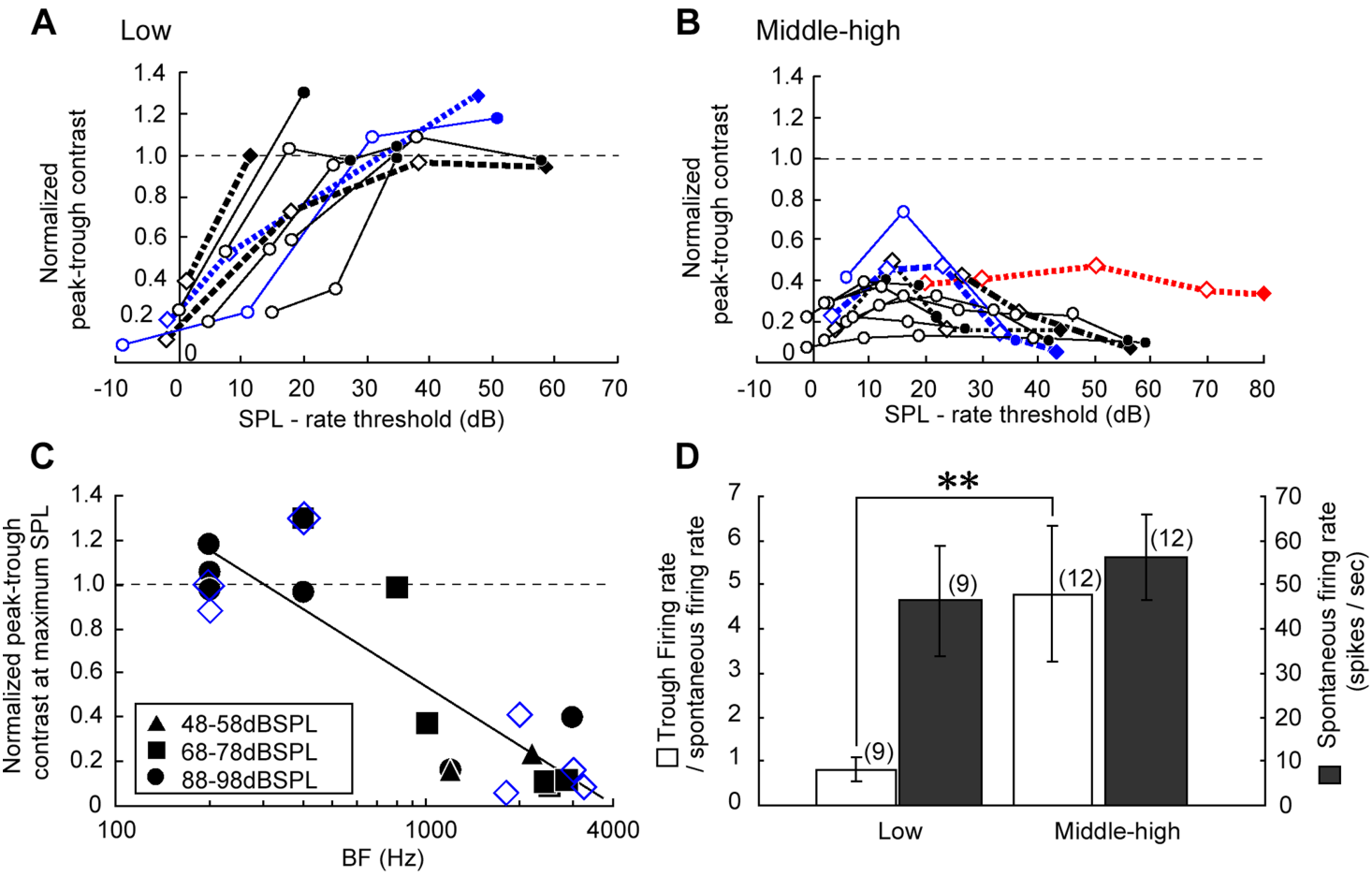

Figure 2. Peak-trough contrast and the firing rate at the worst ITD. $A, B$, Peak-trough contrasts as a function of stimulus intensity relative to the rate threshold. $\boldsymbol{A}$ is for low-BF, and $\boldsymbol{B}$ is for middle-high-BF units, normalized by the maximum firing rate after subtraction of the spontaneous firing rate. The connected symbols indicate the same unit. The filled symbols indicate the maximum SPL applied to the unit. The broken lines connecting diamonds indicate units from young adult chickens. The colors indicate units shown in Figure $1, C(\boldsymbol{A}$, blue solid line), $D(\boldsymbol{A}$, blue broken line), $\boldsymbol{H}$ ( $\boldsymbol{B}$, blue solid line), I ( $\boldsymbol{B}$, blue broken line), and $J$ ( $\boldsymbol{B}$, red broken line). $\boldsymbol{C}$, Peak-trough contrasts evaluated at the maximum SPL for units in $\boldsymbol{A}$ and $\boldsymbol{B}$ after normalization (filled symbols in $\boldsymbol{A}, \boldsymbol{B}$ ). $y=-0.38 \times \log (\mathrm{BF})+3.13 ; r=0.87 ;{ }^{* *} p<0.01 ; n=21$ (14 from juvenile and 7 from young adult). The different symbols indicate different SPLs. The data from young adult chickens were plotted by diamonds hereafter. D, Spontaneous firing rate (filled columns), and firing rates at the worst ITD after normalization by the spontaneous firing rate (open columns) at SPL of the best peak-trough contrast. Error bars indicate SEM.

presence of some inhibition when the stimulus intensity was high. A contribution of GABAergic inhibition has been proposed in the coincidence detection in NL (Hyson et al., 1995; Peña et al., 1996; Funabiki et al., 1998).

\section{Robust SON projection and VGAT immunoreactivity in low-BF region}

The SON is the most likely source of inhibitory inputs to the NL. The SON receives inputs from both the NA and the NL, and forms a GABAergic projection to the ipsilateral NL (Lachica et al., 1994; Yang et al., 1999; Burger et al., 2005). Because NA extracts sound intensity information (Sullivan and Konishi, 1984; Warchol and Dallos, 1990; Köppl and Carr, 2003), the SON projection is predicted to control NL activity in some feedback manner depending on sound intensity (Peña et al., 1996; Burger et al., 2005). We first confirmed the projection from SON to NL by injecting RFP solution into the SON in P3-P7 juvenile chickens $(n=4)$. Figure $3 A-C$ shows the low-power fluorescence images of NL ipsilateral to the injection site at three different rostrocaudal levels. Toward the lateral edge of NL (representing low BF), the intensity and the area of SON projection were expanded. This may reflect the increased number of neurons and the extensive dendritic arbor of individual neurons in the low-BF region (Smith and Rubel, 1979; Kuba et al., 2005). An SON projection to NM was also demonstrated (Fig. 3A).

Distribution of VGAT immunoreactivity overlapped with the SON projection and expanded toward the low-BF region (Fig. $3 D-F)$. Some VGAT-positive particles were densely clustered at the outlines of the cell soma. The number of VGAT-positive particles per region of interest was significantly larger in the low-BF region than in the middle- and high-BF regions (Fig. $3 G$ ). The number of NL cells increased toward the low-BF region (Fig. $3 \mathrm{H}$ ); nevertheless, the density of VGAT-positive particles per cell was significantly higher in the low-BF NL cells (Fig. 3I). The dense distribution in the low-BF NL region of VGAT-positive particles was similarly found in the young adult chicken (P23) (supplemental Fig. 1, available at www.jneurosci.org as supplemental material), and the distribution was not different statistically from that of the juvenile chicken $(p=0.68$; particle number/cell).

RFP-positive SON projections also had many boutons surrounding the cell soma (Fig. $4 B-D$ ) and some extended along the dendrites, particularly in the low-BF cells (Fig. 4A). Most of these boutons were immunoreactive to VGAT as follows (Fig. $4 E-G$ ): $89 \%$ (658 of 739) in low BF, 93\% (317 of 341) in middle BF, and $84 \%$ (103 of 123) in high BF. Therefore, these RFP-positive boutons were most likely GABAergic terminals. These observations and previous studies (Lachica et al., 1994; Yang et al., 1999; Burger et al., 2005) suggest that the SON projections could be responsible for the observed suppression of unit activity in low-BF NL units (Figs. 1, 2).

\section{SON lesion reduced the peak-trough contrast of ITD tuning in low-BF neuron}

We examined how SON contributed to ITD tuning by electrolytic lesions of the ipsilateral SON in juvenile chickens (supplemental Fig. 2, available at www.jneurosci.org as supplemental material). Even after SON lesions, firing rates at the best ITDs were not apparently different from the control; a strong sound stimulus was applied both in the low-BF unit (400 Hz; $78 \mathrm{~dB}$ SPL) (Fig. 5A) 

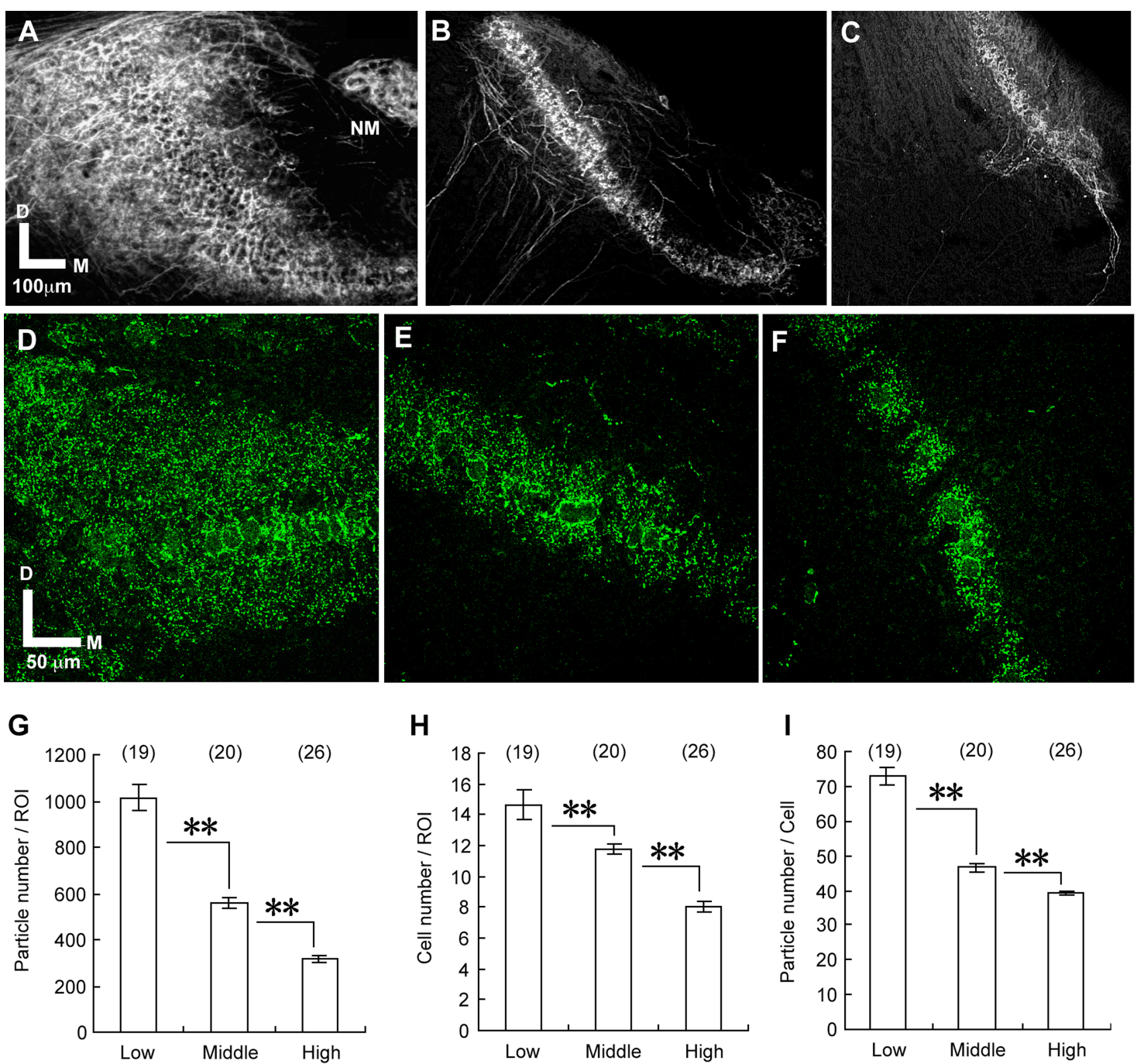

Figure 3. Distribution of SON projection and VGAT immunoreactivity. A-C, Projections from SON to NL visualized by RFP. D, Dorsal; M, medial. D-F, VGAT immunoreactivity. $\boldsymbol{A}, \mathbf{D}$, Low-BF; $\boldsymbol{B}, \boldsymbol{E}$, middle-BF; $\boldsymbol{C}, \boldsymbol{F}$, high-BF region. BF region was determined in reference to Rubel and Parks (1975). $\mathbf{G}-\boldsymbol{H}$, VGAT-positive particles and cells within a region of interest (ROI) $(43.6 \times 53.4 \mu \mathrm{m})$. $\boldsymbol{I}$, Number of VGAT-positive particles per cell. Mean VGAT-positive particle size was $1.31 \pm 0.05 \mu \mathrm{m}^{2}$. Numbers in parentheses are the numbers of regions of interest in which particles were counted in $\mathbf{G}-\mathbf{I}$. Data in Figures 3 and 4 were obtained from juvenile chickens. Error bars indicate SEM. ${ }^{* *} p<0.01$.

and in the middle-high-BF unit (2200 Hz; $68 \mathrm{~dB} \mathrm{SPL})$ (Fig. 5E). However, SON lesions modified the firing activity at the worst ITD in low-BF units (Fig. 5B); the firings at the worst ITD were stable during stimulation and were suppressed only to the level of $0.6 \pm 0.07$ (Fig. 5I) (see the evaluation of suppression in Materials and Methods). This level of suppression was still greater than that of the best ITD $(0.82 \pm 0.03 ; p<0.05)$, but was significantly weaker than that of the worst ITD in the control $(0.09 \pm 0.04 ; p<$ $0.01)$. The suppression was not affected in the middle-high-BF units after SON lesion (Fig. 5I).

The ITD-tuning curves of low-BF units were markedly affected by the SON lesion (Fig. $5 C$ ). When strong sound stimuli were applied, the whole ITD-tuning curve was displaced upward as if it were obtained from the middle-high-BF units in the control. The peak-trough contrast of low-BF units reached the maximum at the sound pressure levels of $\sim 20-50 \mathrm{~dB}$ above the rate threshold and decreased at higher intensities (Fig. 5D). These relative sound intensities needed to attain the maximum peaktrough contrast were comparable with those of the control units (Fig. 2A); however, the absolute levels were lower after SON lesion than in the control because SON lesion reduced the rate threshold of low-BF units $[56.4 \pm 5.6 \mathrm{~dB}$ SPL in the control $(n=$ $6)$ and $25.5 \pm 4.1 \mathrm{~dB}$ SPL after SON lesion $(n=7)$ in low-BF units; $p<0.01$ ] (Fig. 6A). The rate threshold was not affected in the middle-high-BF units $(n=6)$. Moreover, SON lesion did not affect either PSTHs, ITD-tuning curves, or the dependence of peak-trough contrast on the sound intensity in the middlehigh-BF units (Fig. 5E-H). Consequently, the peak-trough contrast obtained with loud sounds became less dependent on the BF after SON lesion than in the control (Fig. $5 J$ ). These observations indicate that inhibition from SON was BF dependent and stronger in the low-BF units than in the middle-high-BF units. SON lesions did not affect the spontaneous firing rate $(p=1)$ or the maximum firing rate at the best ITD $(p=0.63)$, regardless of $\mathrm{BF}$. Phase-locking (Rayleigh test; $p<0.05$ ) at the best ITD was BF dependent and was not affected by the SON lesion $(p>0.15)$ (Fig. $6 B$ ). The size of SON lesion did not have any correlation with the lesion effects; for example, the level of peak-trough contrast at the strong SPL was not correlated (coefficient of determination, $r^{2}=0.002 ; n=6$ low-BF units) (see details in the legend of supplemental Fig. 2, available at www.jneurosci.org as supplemental material). 

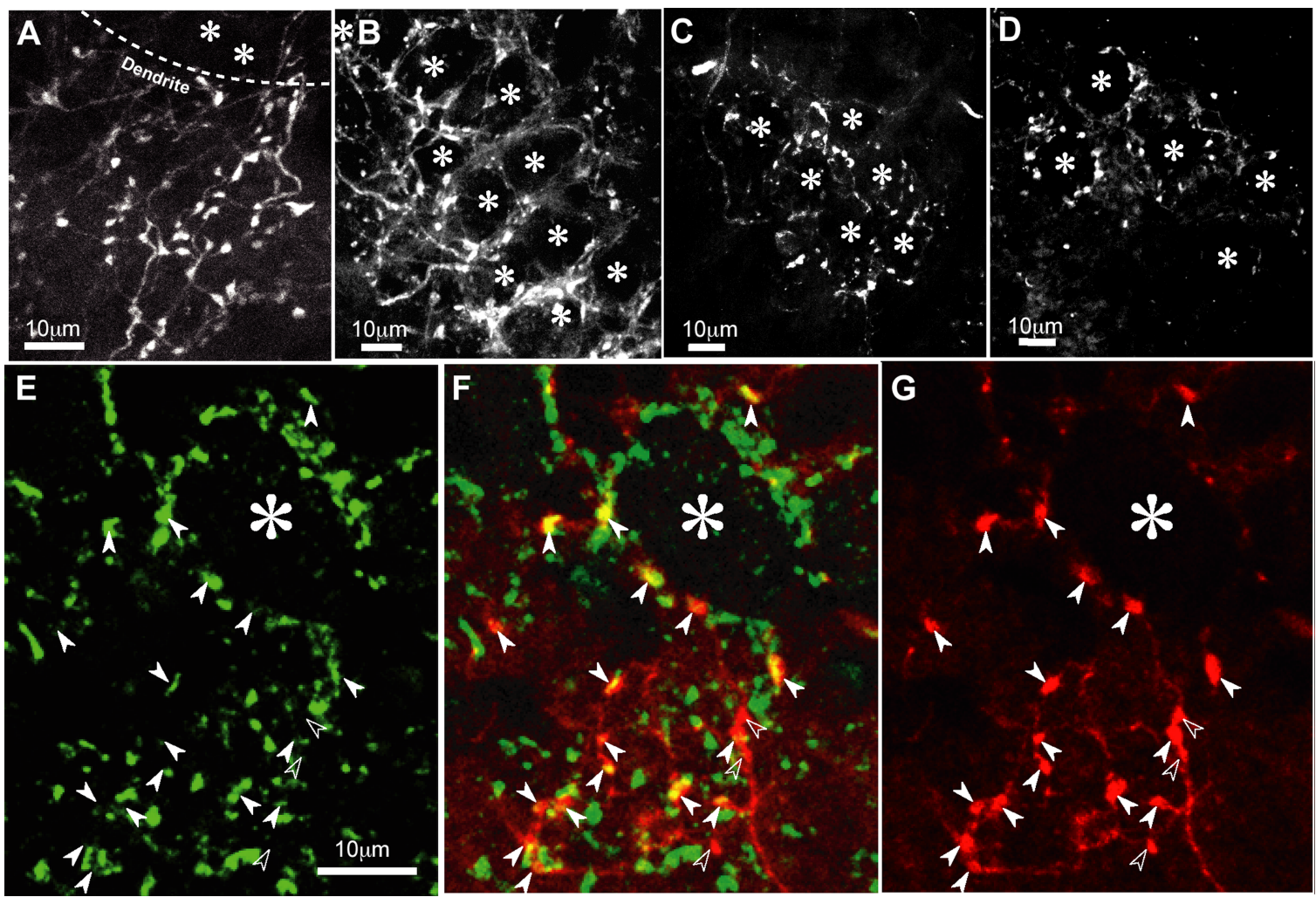

Figure 4. Immunostaining with VGAT and RFP projection from SON. $A-D$, SON projections to NL at high magnification. The asterisks indicate cell somas. The broken line in $A$ demarcates the area of cell somas from dendrites. $\boldsymbol{A}, \boldsymbol{B}$, Low-BF; $\boldsymbol{C}$, middle-BF; $\boldsymbol{D}$, high-BF region; $\boldsymbol{E}$, VGAT; $\boldsymbol{F}$, merged image; $\boldsymbol{G}$, SON projection. The double-labeled particles are indicated by filled arrowheads and RFP-positive but VGAT-negative particles by open arrowheads; these may be an axon and a cut end of it.

Responsiveness to ITD enhanced by the inhibition from SON Figure $7 A$ shows a part of ITD-tuning curves of the best peaktrough contrast from juvenile units and compares the control with those after the SON lesion (two units from Figure 1 for the control and two after the SON lesion in Figure 5). They were normalized by the maximum firing rate of each unit. The responsiveness to ITD was evaluated as the maximum difference of normalized firing rate within the physiologically relevant ITD $( \pm 100$ $\mu$ s; shaded area) in the tuning curve (see Materials and Methods). A small number of low-BF units (one of six) and one-half of the middle-high-BF units (four of eight) had a peak or a trough of the ITD-tuning curve within this range of ITD in the control, and this tendency was not affected by the SON lesion (two of seven in low $\mathrm{BF}$; three of six in middle-high $\mathrm{BF}$ ). After the SON lesion, the responsiveness to ITD was smaller both in the low-BF unit (red solid line) and in the middle-high-BF unit (red broken line) than in the corresponding control unit (blue lines). Figure $7 B$ shows all the measured responsiveness to ITD. The triangles indicate the units shown in Figure $7 A$; the responsiveness of low-BF unit in Figure $7 A(200 \mathrm{~Hz})$ was the smallest in the control units, and that of middle-high-BF unit $(2400 \mathrm{~Hz})$ was the largest.

The responsiveness to ITD, measured within the fixed ITD, depended on BF and was smaller in low-BF units; this is because ITD-tuning curves have steeper slopes for the higher-BF units and shallower slopes for the lower-BF units as a physical consequence of sound frequency. This tendency was enhanced after SON lesion (red; $n=13$ ). On average, the responsiveness to ITD after SON lesion became significantly smaller in low-BF units $(6.5 \pm 1.7 \% ; n=7)$ than in middle-high-BF units $(16.4 \pm 2.8 \%$; $n=6)$ (Fig. $7 C)(p<0.01)$. In contrast, the responsiveness to ITD in the control was not significantly different among $\mathrm{BF}$ in the juvenile chicken: $17.5 \pm 4.4 \%(n=6)$ in low-BF units and $19.4 \pm$ $3.6 \%(n=8)$ in middle-high-BF units (Fig. $7 C)(p=0.38)$. The responsiveness was not significantly different statistically $(p=$ 0.14 ) either when evaluated by the absolute firing rate (supplemental Fig. 3, available at www.jneurosci.org as supplemental material).

Inhibition by SON improved other parameters of ITD-tuning curves, such as the half-peak width, the maximum slope, and the maximum difference between peak-trough firing rates. When compared regardless of the physiologically relevant ITD that was determined by the head size of the animal, the half-peak width normalized to the half-period of BF was significantly smaller in the control low-BF units than after SON lesion $(0.87 \pm 0.06, n=$ 11 in the control; $1.10 \pm 0.04, n=10$ after SON lesion; $p=$ $0.004)$. The maximum slope was significantly reduced after SON lesion in low-BF units $[0.41 \pm 0.08$ in the control and $0.24 \pm 0.08$ (firing rate/ $\mu \mathrm{s}$ ) after SON lesion; $p=0.02]$, despite the fact that the maximum slope was smaller in lower-BF units, as a physical consequence of sound frequency. The maximum difference between the peak-trough firing rates was smaller after the SON lesion in the low-BF units $[227.13 \pm 26.82$ in the control and $110.39 \pm 27.15$ (spikes/s) after SON lesion; $p=0.003$ ] (Fig. 5J). Middle-high-BF units were not affected $(p>0.2)$.

Seven measures of responsiveness to ITD made in the control young adult chickens were plotted in Figure $7 B$ with diamonds (blue). Although sample numbers were small, the distribution was not particularly different from that of juvenile chickens in the low-BF units $(p=0.25)$. Two of three low-BF units had either peak or trough within the $\pm 100 \mu$ s ITD window, whereas among four middle-high-BF units, one had a trough and another had both peak and trough. The responsiveness was slightly higher in 
the young adult than in the juvenile in the middle-high-BF units $(p=0.04)$ (Fig. $7 C)$. The difference between the low- and the middle-high-BF units was also significant $(p=0.02)$.

A topographical distribution of ITD tuning within NL should affect the responsiveness to ITD that measured within the narrow and fixed window of ITD. Despite the narrowness of the ITD window, some middle-high-BF units of NL had both the peak and the trough within it, which maximized the responsiveness to ITD. In contrast, the peak ITD located outside the physiologically relevant ITD increased the dynamic range within the physiological range in the low-BF MSO neurons of gerbils (Brand et al., 2002). We do not have appropriate data to evaluate the topographical mapping of ITD tuning within NL. In the barn owl, the ITD tuning property was topographically mapped within $\mathrm{NL}(\mathrm{BF}>3 \mathrm{kHz})$ (Carr and Boudreau, 1991). A similar topographical representation was observed in the chicken NL at the $\mathrm{BF}$ region $>800 \mathrm{~Hz}$; however, at the lower-BF region, the topographical mapping remains still uncertain (Köppl and Carr, 2008).

\section{Phase distribution of firing activities in ITD tuning}

The number of spikes was modulated with ITD (Fig. $8 A$, right top), and the firing activity of this low-BF unit was distributed within a narrow phase when plotted in a period histogram (Fig. $8 \mathrm{~A}$, left). At the worst ITD, the number of spikes was extremely reduced, and the phase locking of the firing activity was not significant (Rayleigh test; $p<0.05$ ). Consequently, the vector strength of unit activities was evaluated only at the limited ITDs and was just $\sim 1.0$ (Fig. $8 \mathrm{~A}$, right middle), and the best phase (right bottom) remained within a narrow phase angle (Fig. $8 \mathrm{~A}$, left bottom plotted all the unit activities regardless of ITDs). This narrow phase distribution seems consistent with the narrow time window that improved the coincidence detection (Kuba et al., 2003).

The narrow phase distribution in the control contrasts with the unit activities after $\mathrm{SON}$ lesion (Fig. $8 \mathrm{~B}$ ). The best phase of unit activities was displaced with ITD and was distributed at angles of $\sim 360^{\circ}$ when all firing activities were plotted in one period histogram (Fig. $8 \mathrm{~B}$, bottom). However, at each ITD, unit activities were still well phase-locked to sound when a strong SPL was applied (Fig. 8 B, left; vector strength in the right middle) (Rayleigh test, $p<0.05$ ). We measured ITD in reference to the contralateral stimulus in this study. Therefore, the phase displacement after SON lesion indicated that unit activities followed the ipsilateral sound stimulus. SON lesion might SEM.
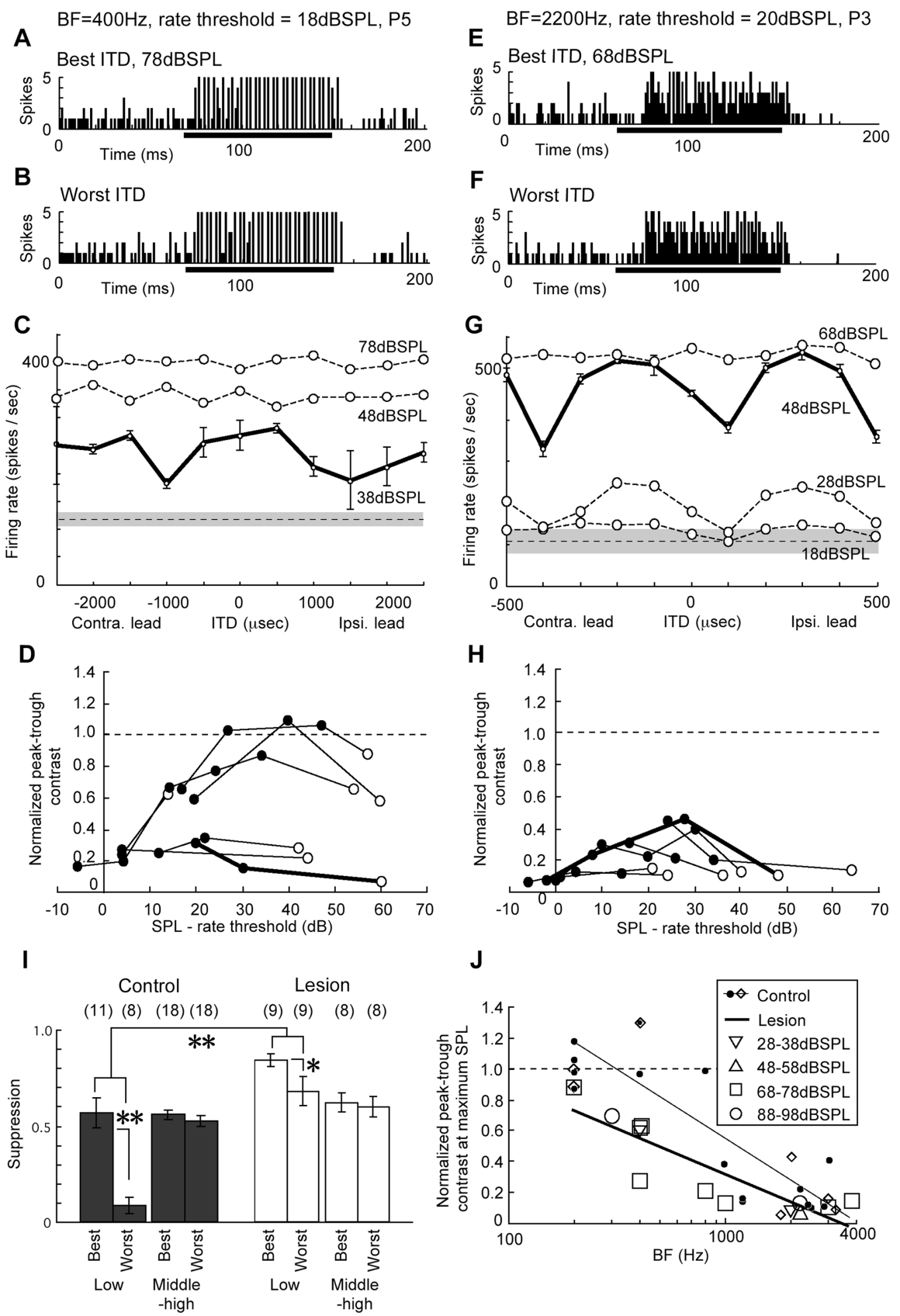

$$
\mathbf{J}
$$

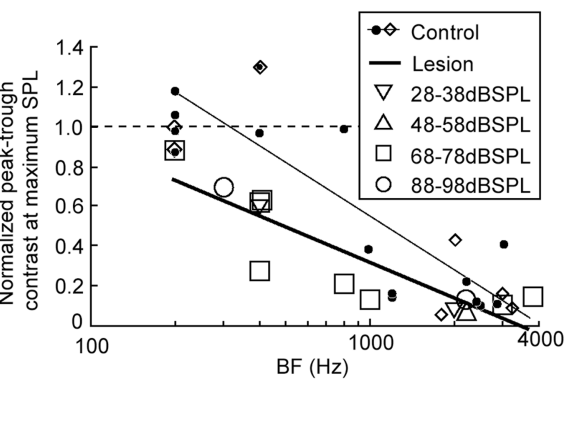

Figure 5. Unit responses after SON lesion in the juvenile chicken. $\boldsymbol{A}-\boldsymbol{C}$, Low-BF units $(400 \mathrm{~Hz})$. $\boldsymbol{E}-\boldsymbol{G}$, Middle-high-BF units $(2200 \mathrm{~Hz})$. A, PSTHs at the best ITD. B, PSTHs at the worst ITD. C, The ITD-tuning curves. $\boldsymbol{E}-\mathbf{G}$ correspond to $\boldsymbol{A}-\boldsymbol{C}$. The error bars are $S D$ in $\boldsymbol{C}(n=2$ repeats). $\boldsymbol{D}, \boldsymbol{H}$, Normalized peak-trough contrasts correspond to Figure 2, $A$ and $B$. The maximum SPL applied to each unit is indicated by open symbols. I, Suppression of spike firing rate at the best and the worst ITD. Some units in the control low BF showed almost no spikes at the worst ITDs and were evaluated only at the best ITDs. J, The normalized peak-trough contrast plotted against BF for units after SON lesion, evaluated at the maximum SPL applied to the unit ( $\boldsymbol{D}, \boldsymbol{H}$, open symbols). The plots for the control units (dots) are the same as in Figure 2C. Different symbols indicate SPL. The thick line is for the SON lesion; $y=-0.25 \times \log (\mathrm{BF})+2.06 ; r=0.88 ;{ }^{*} p<0.05 ;{ }^{* *} p<0.01 ; n=13$; and the thin line is for the control. Error bars indicate

have made the input from the ipsilateral NM stronger and unbalanced. Consistently, the inhibitory effects of SON stimulation or GABA application were observed on the firing activity of NM neurons in slices (Monsivais et al., 2000; Lu and Trussell, 2001).

The imbalance of excitatory inputs may be correlated with the different levels of peak-trough contrast among units after SON lesion (Fig. 5D and related discussion in Fig. 9D) in which some units had a peak-trough contrast even $>1$ at the intermediate 
A

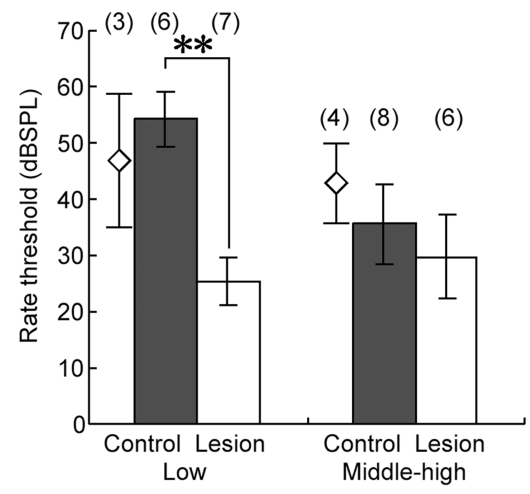

B

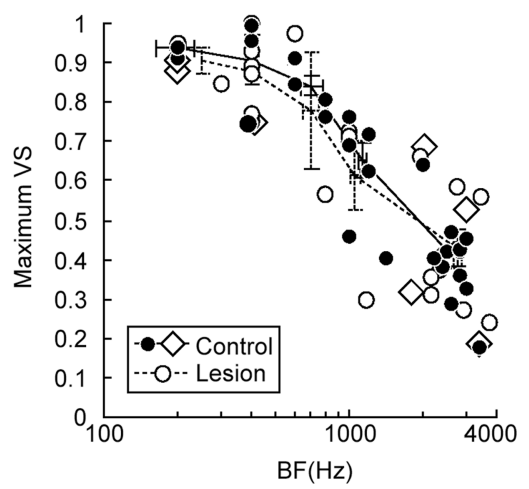

Figure 6. Rate threshold and vector strength. $A$, Rate threshold of units in control and after SON lesion. In low-BF neurons, the rate threshold was reduced significantly after SON lesion. The numbers in parentheses are the numbers of units, and ${ }^{* *}$ indicates the statistical significance of $p<0.01$ ( $t$ test). The diamonds indicate the mean from young adult chickens. Rate thresholds were not different in the control between the young adult and the juvenile chickens: $p=0.28$ for low-BF and $p=0.23$ for middlehigh-BF units. $\boldsymbol{B}$, VS from all units recorded. VSs were calculated from unit activities at the best ITD at the maximum SPL applied in each unit when phase-locking was significant (Rayleigh test; $p<0.05$ ) and were not different between the control and the units after SON lesion ( $t$ test; $p>0.15$ for all BF range). Bin width for statistics was set as follows: $<300,400,600-800$, $1000-1600$, and $>2000 \mathrm{~Hz}$. Error bars indicate SEM.

SPLs, and the peak-trough contrast decreased slightly at higher SPLs. Nevertheless, the phase displacement occurred as a function of ITD in all low-BF units after SON lesion ( $n=10$ units). Therefore, the different levels of peak-trough contrast might be a consequence of some imbalance in NM activities after lesion of SON (Fig. 9D).

\section{Computer simulation of ITD tuning}

The effects of SON inhibition on ITD tuning in NL were further examined with computer simulation (see Materials and Methods). The SON inhibition was incorporated as (1) the inhibitory conductance in NL and (2) the balanced excitatory synaptic inputs from both sides of NM. The imbalance of bilateral excitatory inputs after the SON lesion were simulated by setting the ipsilateral conductance at twice the size of the contralateral conductance, while the average conductance of bilateral EPSPs was maintained at the same level as the control (Fig. 9). The simulation demonstrated a well phase-locked firing activity when bilateral stimuli were applied in-phase, regardless of SON inhibition (Fig. 9A, top). However, the absence of SON inhibition produced the phase displacement as a function of ITD (Fig. 9A, bottom right). This phase displacement disappeared when the bilateral excitatory inputs were of an equal strength (data not shown).

The size and the time course of EPSPs were significantly affected by the SON inhibition: the amplitude increased 2.8 times, and the half-peak width increased 1.4 times in the absence of SON inhibition (Fig. 9B). The firing rate at the best ITD increased with the excitatory inputs regardless of SON inhibition (Fig. 9C, 0 and $360^{\circ}$ ); in contrast, the firing rate at the worst ITD decreased only in the presence of inhibition (Fig. $9 \mathrm{C}$, blue, $180^{\circ}$ ). This inhibition enhanced the peak-trough contrast as a function of excitatory input (Fig. 9D, blue). In contrast, the firing rate at the worst ITD increased as a function of excitatory inputs without SON inhibition, and became almost the same as that of the best ITD when the excitatory conductance was large (Fig. 9C, red). This reduced the peak-trough contrast at the strong excitatory inputs (Fig. 9D, red). The simulation further demonstrated that the peak-trough contrast without inhibitory conductance to NL was critically dependent on the balance of bilateral excitatory

inputs and was significantly reduced by the loss of balance (Fig. 9D, red). They indicate that SON activities increase the peaktrough contrast of ITD tuning in NL differentially: first by accelerating the EPSP time course, and second by balancing the bilateral excitatory inputs, through the sound-intensity-dependent feedback inhibition on NL and NM, respectively.

\section{Discussion}

We found that the firing activities in the control low-BF units were affected robustly by ITD and were suppressed at the worst ITD by loud sound (Fig. 1). This enhanced the peak-trough contrast of ITD tuning. The contrast was dependent on the intensity and the frequency of sound, and was enhanced in the low-BF units by SON activity (Figs. 5, 7), both through narrowing the time window for coincidence detection in NL represented as the narrowness of phase distribution (Fig. 8) and through balancing the excitatory inputs from each NM (Fig. 9). Consequently, the feedback inhibition from SON enhanced the peak-trough contrast and compensated for the small ITD cue in a soundintensity-dependent manner.

The inhibition from SON is particularly important in the low-BF NL units for precise ITD coding. The period of ITDtuning curve is determined by the BF of the unit, and the slope of ITD-tuning curve is shallower in the lower-BF units. This will reduce the change of firing rate within a narrow range of ITD (responsiveness to ITD) (Fig. 7A). Suppression of the firing rate at the worst ITD by SON activity may counterbalance this shallowness of the ITD-tuning curve significantly. Consequently, the peak-trough contrast is enhanced in the low-BF units (Fig. 2C). This inhibition from SON is, however, not so strong as to suppress the neural activity completely at the best ITD, which suggests that the level of inhibition might be precisely regulated. In combination with the sound intensity information from NA, the SON may use NL signals as a control to optimize the level of feedback inhibition in both NL and NM.

The importance of feedback inhibition from SON has been indicated by an investigation of projection pattern of SON (Burger et al., 2005) and by a network model (Dasika et al., 2005). They proposed that the projection to the contralateral SON was essential in balancing the excitability of NM, particularly when the sound source is lateralized (Burger et al., 2005). By simulating a network model including NM, NA, NL, and SON, Dasika et al. (2005) have concluded that inhibitory feedback substantially increases the sensitivity of ITD coding by maintaining the balanced inputs to NL. Peña et al. (1996) have proposed a gain-control activity of SON to make the ITD tuning of NL units of barn owl tolerant to loud sound. Balancing the firing activity of NA and NM through reciprocal networks between SON and NA, and bilateral SONs may be crucial in the operation of regulatory networks. Moreover, binaural sound intensity and phase are expected to interfere with each other through the acoustic interference across the interaural canal (Hyson et al., 1995), which we have not addressed in this study. To understand how these balancing mechanisms operate in processing the interaural differences of sound information, additional experiments are required. 
A

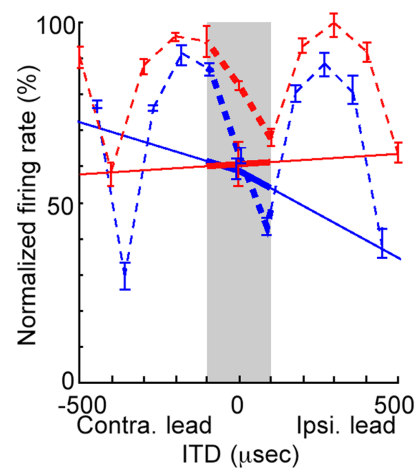

B

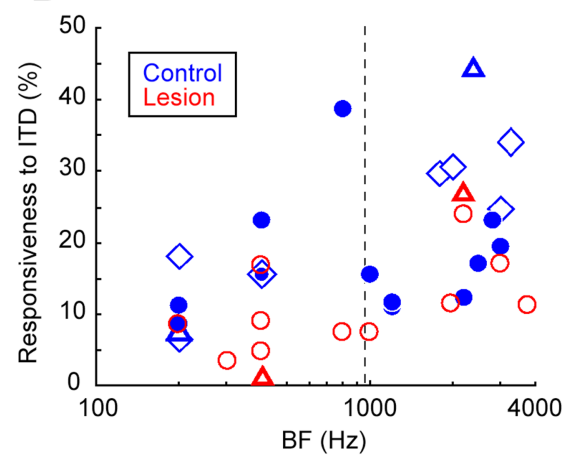

C

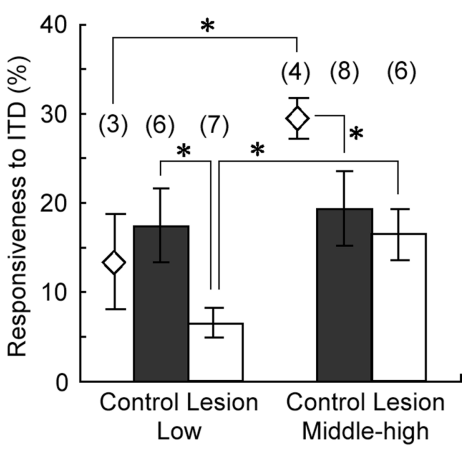

Figure 7. Responsiveness to ITD and the effects of SON lesion. $A$, ITD-tuning curves of best contrast for four units shown in Figures $1, C$ and $H$, and $5, C$ and $G$, for ITD of $\pm 500 \mu$ s. The firing rate was normalized by the maximum firing rate of each unit. The broken lines are for middle-high-BF units (BF $\geq 1 \mathrm{kHz}$ ), and the solid lines are for low-BF units ( $B F<1 \mathrm{kHz}$ ). Controls are in blue, and after SON lesions are in red in $\boldsymbol{A}$ and $\boldsymbol{B}$. The shaded area indicates $\pm 100 \mu$ s ITD to measure the responsiveness. Contra., Contralateral; Ipsi., ipsilateral. $\boldsymbol{B}$, Responsiveness to ITD from units in Figures $2, A$ and $B$, and $5, D$ and $H$ ( 27 units from the juvenile chickens for both control and SON lesion and 7 units from the young adult chickens for control). The triangles are from the units in $A$. The vertical dashed line at $1 \mathrm{kHz}$ indicates the boundary between the low and middle-high BF. C, Mean responsiveness to ITD for units in $\boldsymbol{B}$. The diamonds indicate the mean from young adult chickens. ${ }^{*} p<$ 0.05. Error bars indicate SEM.

A Control

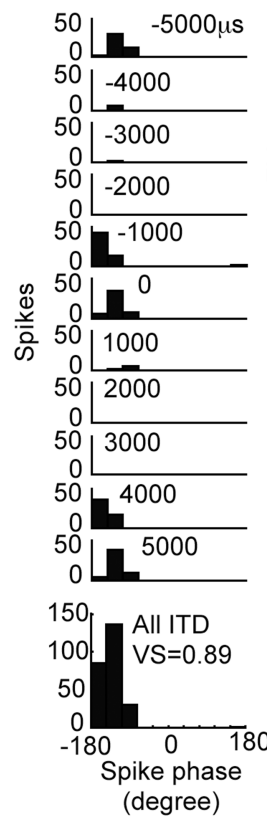

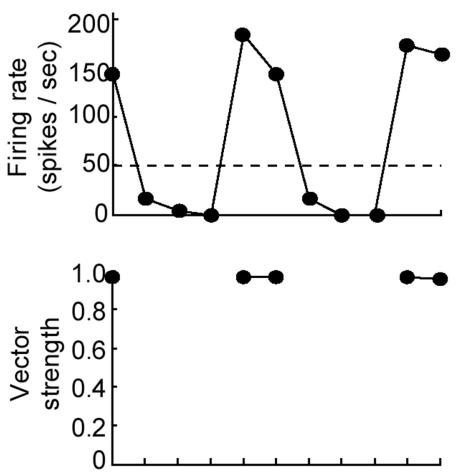

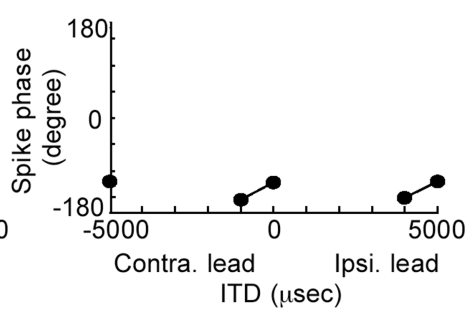

B

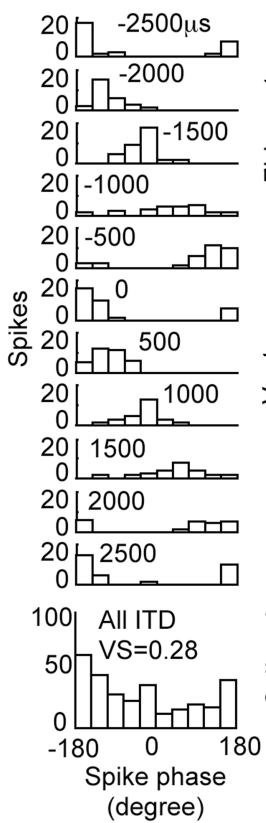

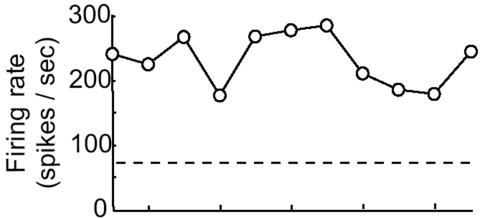
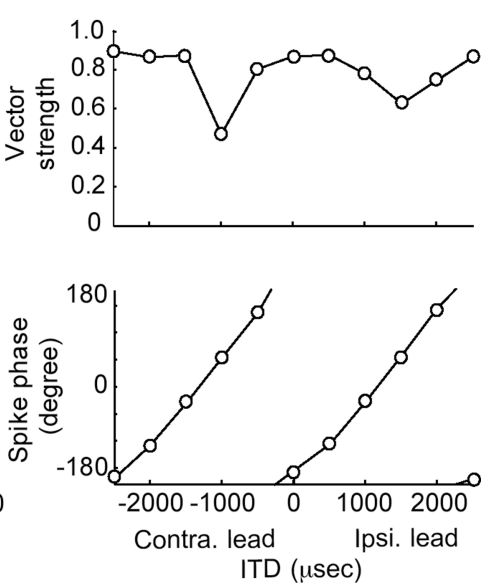

Figure 8. Sound-phase dependency of low-BF unit activity. $\boldsymbol{A}, \boldsymbol{B}$, Period histograms (left), ITD-tuning curve, vector strength, and the best phase (right, from the top to the bottom) for a control in $\boldsymbol{A}$ and after SON lesion in $\boldsymbol{B}$. $\boldsymbol{A}$ and $\boldsymbol{B}$ are the units shown in Figures $1 \mathrm{C}(98 \mathrm{~dB}$ SPL; BF, $200 \mathrm{~Hz})$ and 5 C (38 dB SPL; BF, $400 \mathrm{~Hz}$ ), respectively, at the SPLs of best peak-trough contrast. A period histogram was calculated at each ITD (indicated in each plot) in reference to the phase of contralateral sound. At the bottom $(\boldsymbol{A}, \boldsymbol{B}$, left), all unit activities were plotted regardless of ITD. Vector strength was calculated when the phase-locked firing was significant (Rayleigh test; $p<0.05$ ). Contra., Contralateral; Ipsi., ipsilateral.

\section{Effects of development}

An increasing gradient of GABA immunoreactivity from the rostromedial (high-BF) to caudolateral (low-BF) region was described by NM, and the gradient was the greatest in the posthatch chicken (P0-P9) (Code et al., 1989). In NL, more GABAimmunopositive terminals were found in the low-BF region than in the other NL regions in the chicken (Code et al., 1989), as well as in the caudolateral region termed lateral laminaris in the barn owl (Carr et al., 1989). GABA-immunoreactive neurons were found surrounding NL and NM; however, most of the immunoreactivity in NL may originate from the projection from the SON (Lachica et al., 1994; Monsivais et al., 2000; Burger et al., 2005).

Neuronal responsiveness to sound in NM and NL changes with development in the chicken (Lippe and Rubel, 1985). Neurons at a given location in NM and NL became maximally responsive or tuned to progressively higher frequencies during development. These developmental changes of neuronal responsiveness to sound might have affected our observation of ITD tuning.

The single-unit recordings in the young adult chickens were more difficult than in the juvenile chickens, because the robust neurophonic responses disturbed the spike isolation. Most analysis was based on 7 NL units in the young adult and 27 units in the juvenile chicken. Sample numbers are still small; however, we did not find any systematic difference of ITD-tuning properties between the juvenile and the young adult chicken. Especially in the 


\section{A}
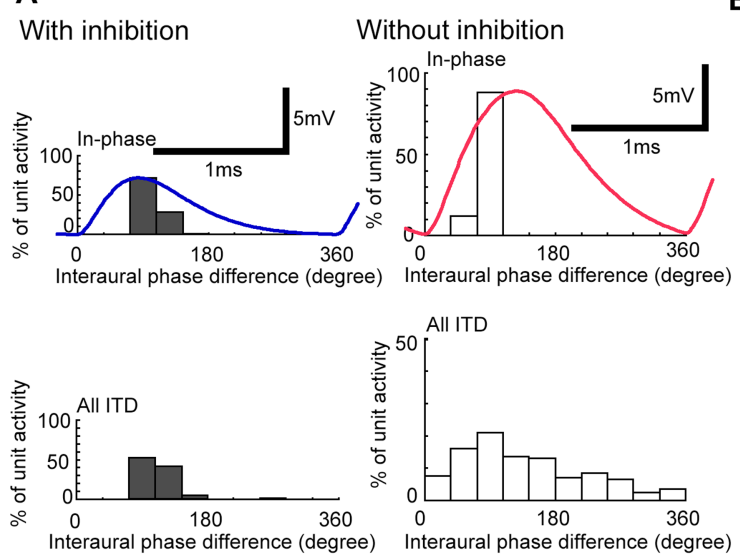

C

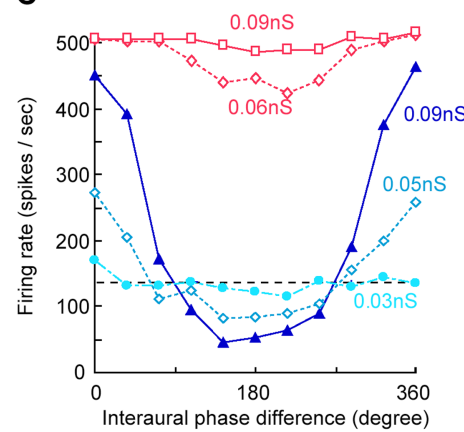

D

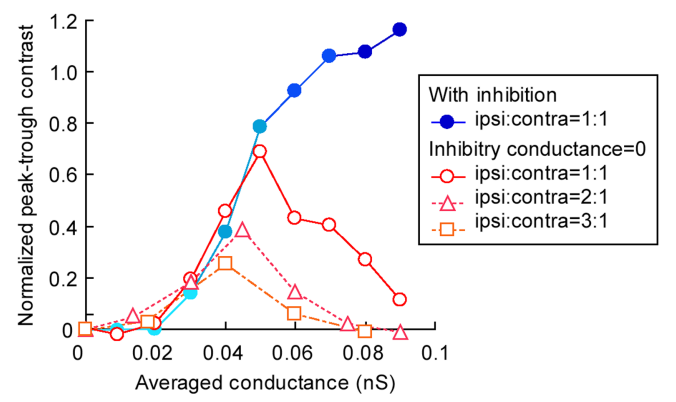

Figure 9. Simulation of EPSPS, period histograms, and ITD-tuning curves. $\boldsymbol{A}$, Period histograms with inhibition (left as a control, shaded column) and without inhibition (right as after SON lesion, open column) were calculated for a unit of BF $500 \mathrm{~Hz}$. At the top, bilateral stimuli were applied in-phase, without ITD. Overlaid EPSPs were calculated at the soma. The histograms at the bottom show all the firing activities regardless of ITD. The averaged conductance for the two EPSCs was $0.09 \mathrm{nS}$ for the calculations. This conductance was selected to mimic the quasi-maximum sound intensity level we applied in the experiments. SON lesion was mimicked by setting the inhibitory conductance to zero, and making the ipsilateral EPSP larger than the contralateral EPSP by a factor of 2; contralateral, $0.06 \mathrm{nS}$, versus ipsilateral, $0.12 \mathrm{nS}$. B, Amplitude (top) and half-peak width of ipsilateral EPSP (bottom) were calculated at the soma from a series of 40 simulations. $C$, ITD-tuning curves were simulated for the control (blue) and for the SON lesion (red) by the same set of parameters in $\boldsymbol{A}$ and plotted as a function of interaural phase difference. Averaged EPSP conductance was indicated. A horizontal broken line indicates the spontaneous firing rate. $\boldsymbol{D}$, Normalized peak-trough contrast as a function of averaged conductance of bilateral excitatory synaptic inputs, when inhibition was included ( ), and inhibition was excluded (open symbols). Different open symbols indicate different levels of imbalance of EPSP conductance, 1:1 for contralateral (contra) to ipsilateral (ipsi), $\bigcirc ; 1: 2, \triangle ; 1: 3, \square$.

low-BF unit, the contrast of ITD tuning was enhanced at the strong SPL in all units investigated (Figs. 1, 2). In the middlehigh-BF units, the contrast of ITD tuning was reduced at the strong SPL and this property was observed in all juvenile units and in three of four young adult units. There were also no differences in the spontaneous firing rate (Fig. 2D), the rate threshold (Fig. 6A), and the vector strength (Fig. 6B). Moreover, the distribution pattern of VGAT-positive terminals was not different [P4 (Fig. 3); P26 (supplemental Fig. 1, available at www. jneurosci.org as supplemental material)].

One middle-high-BF unit from a young adult chicken demonstrated a tolerance of ITD tuning to SPL (Figs. $1 E, J, 2 B$ ) and appeared similar to the observation made in the adult barn owl NL unit (Peña et al., 1996). Although the firing rate at the worst ITD of this unit was still far higher than the spontaneous rate, this may reflect that the inhibition from SON may develop with maturation. The ITD sensitivity in the middle-high-BF units might also be increased during maturation (Fig. $7 C)(p=0.04)$. How extensively the neuronal responsiveness develops after hatching deserves additional examination, including the activities in SON and the effects of inhibition from SON, not only in NL but also in NA and NM.

Both mammals and birds overcome the small interaural time difference cues by using the sound intensity cues

Involvement of inhibition in ITD tuning in MSO was reported in some mammalian species. In dogs (Goldberg and Brown, 1969) and cats (Yin and Chan, 1990), the firing rate of MSO units at the worst ITD was lower than that of monaural stimulation and sometimes even lower than the spontaneous firing rate. MSO receives inhibitory projections from lateral and medial nucleus of trapezoid body (LNTB and MNTB), both of which receive input from the globular bushy cell in the anterior ventral cochlear nucleus (AVCN) (Cant and Hyson, 1992). The firing activity of MNTB is tightly phase-locked to the contralateral sound (Smith et al., 1998; Tollin and Yin, 2005), implying a crucial role of precisely timed inhibition in ITD tuning in MSO. In gerbils, this phase-locked inhibition is proposed to play a pivotal role in ITD tuning of low-frequency sound (Brand et al., 2002). This is based on the observation that the best ITD was consistently at the contralateral leading ITD outside of the physiologically relevant ITD and shifted to 0 after blocking glycinergic inhibition from MNTB. The inhibition from MNTB displaces the best ITD outside of, brings the slope of ITD-tuning curve within, and makes the contrast of ITD tuning greater within the physiological range of ITD (Grothe, 2003). From these observations, it is proposed that ITD tuning in the gerbil MSO is the product of an interaction of precisely timed inhibitory and excitatory inputs. However, some concerns about the interpretation of the inhibitory effects have been presented (Joris and Yin, 2007). A different mechanism of inhibition was proposed to displace the best ITD outside of the physiologically relevant ITD, based on the anatomical observations of asymmetrical cell structure of generating the axon from the dendrite that receives ipsilateral inputs; by the interplay between the $\mathrm{Na}^{+}$channel activity with glycine-mediated inhibitory inputs, a fine mechanism for the adjustment of the best ITD has been proposed (Zhou et al., 2005). Alternative mechanisms to create the "internal delay" to explain the ITD coding in mammals have been discussed in detail previously (Joris and Yin, 2007).

The MNTB provides inhibitory inputs to the lateral superior olive where interaural level difference is processed between the ipsilateral excitatory input from AVCN. Therefore, the sound information carried by the MNTB neuron could be a function of SPL and the inhibitory synapse onto MSO may modulate the ITD processing in an SPL-dependent manner. Ipsilateral inhibitory innervation to MSO is from LNTB and is also dependent on SPL, because LNTB receives input from the globular bushy cells similar to MNTB (Smith et al., 1991). Both mammals and birds appear to use sound intensity information in processing sound 
timing information, to compensate for the small ITD cues. They appear to pursue different strategies, namely, the feedforward inhibition adopted in mammals and the feedback inhibition adopted in birds (Brand et al., 2002; Grothe, 2003; Harper and McAlpine, 2004; Burger et al., 2005). Feedforward inhibition may have an advantage in speed and simple network structure over the feedback inhibition, although the feedback inhibition in birds may be more stable and may be appropriate for controlling the fine-tuning of ITD detection for low-frequency sound.

\section{References}

Brand A, Behrend O, Marquardt T, McAlpine D, Grothe B (2002) Precise inhibition is essential for microsecond interaural time difference coding. Nature 417:543-547.

Burger RM, Cramer KS, Pfeiffer JD, Rubel EW (2005) Avian superior olivary nucleus provides divergent inhibitory input to parallel auditory pathways. J Comp Neurol 481:6-18.

Cant NB, Hyson RL (1992) Projections from the lateral nucleus of the trapezoid body to the medial superior olivary nucleus in the gerbil. Hear Res 58:26-34.

Carr CE, Boudreau RE (1991) Central projections of auditory nerve fibers in the barn owl. J Comp Neurol 314:306-318.

Carr CE, Fujita I, Konishi M (1989) Distribution of GABAergic neurons and terminals in the auditory system of the barn owl. J Comp Neurol 286:190-207.

Code RA, Burd GD, Rubel EW (1989) Development of GABA immunoreactivity in brainstem auditory nuclei of the chick: ontogeny of gradients in terminal staining. J Comp Neurol 284:504-518.

Dasika VK, White JA, Carney LH, Colburn HS (2005) Effects of inhibitory feedback in a network model of avian brain stem. J Neurophysiol 94:400-414.

Fukui I, Sato T, Ohmori H (2006) Improvement of phase information at low sound frequency in nucleus magnocellularis of the chicken. J Neurophysiol 96:633-641.

Funabiki K, Koyano K, Ohmori H (1998) The role of GABAergic inputs for coincidence detection in the neurones of nucleus laminaris of the chick. J Physiol 508:851-869.

Furuta T, Tomioka R, Taki K, Nakamura K, Tamamaki N, Kaneko T (2001) In vivo transduction of central neurons using recombinant Sindbis virus: Golgi-like labeling of dendrites and axons with membrane-targeted fluorescent proteins. J Histochem Cytochem 49:1497-1508.

Goldberg JM, Brown PB (1969) Response of binaural neurons of dog superior olivary complex to dichotic tonal stimuli: some physiological mechanisms of sound localization. J Neurophysiol 32:613-636.

Grothe B (2000) The evolution of temporal processing in the medial superior olive, an auditory brainstem structure. Prog Neurobiol 61:581-610.

Grothe B (2003) New roles for synaptic inhibition in sound localization. Nat Rev Neurosci 4:540-550.

Harper NS, McAlpine D (2004) Optimal neural population coding of an auditory spatial cue. Nature 430:682-686.

Hyson RL, Overholt EM, Lippe WR (1994) Cochlear microphonic measurements of interaural time differences in the chick. Hear Res 81:109-118.

Hyson RL, Reyes AD, Rubel EW (1995) A depolarizing inhibitory response to GABA in brainstem auditory neurons of the chick. Brain Res 677:117-126.

Joris P, Yin TC (2007) A matter of time: internal delays in binaural processing. Trends Neurosci 30:70-78.
Klump G (2000) Sound localization in birds. In: Comparative hearing: birds and reptiles (Dooling RJ, Fay RR, Popper AN, eds). New York: Springer.

Konishi M (2003) Coding of auditory space. Annu Rev Neurosci 26:31-55.

Köppl C, Carr CE (2003) Computational diversity in the cochlear nucleus angularis of the barn owl. J Neurophysiol 89:2313-2329.

Köppl C, Carr CE (2008) Maps of interaural time difference in the chicken's brainstem nucleus laminaris. Biol Cybernetics 98:541-559.

Kuba H, Yamada R, Ohmori H (2003) Evaluation of the limiting acuity of coincidence detection in nucleus laminaris of the chicken. J Physiol 552:611-620.

Kuba H, Yamada R, Fukui I, Ohmori H (2005) Tonotopic specialization of auditory coincidence detection in nucleus laminaris of the chick. J Neurosci 25:1924-1934.

Kuba H, Ishii TM, Ohmori H (2006) Axonal site of spike initiation enhances auditory coincidence detection. Nature 444:1069-1072.

Lachica EA, Rübsamen R, Rubel EW (1994) GABAergic terminals in nucleus magnocellularis and laminaris originate from the superior olivary nucleus. J Comp Neurol 348:403-418.

Lippe W, Rubel EW (1985) Ontogeny of tonotopic organization of brain stem auditory nuclei in the chicken: implications for development of the place principle. J Comp Neurol 237:273-289.

Lu T, Trussell LO (2001) Mixed excitatory and inhibitory GABA-mediated transmission in chick cochlear nucleus. J Physiol 535:125-131.

Monsivais P, Yang L, Rubel EW (2000) GABAergic inhibition in nucleus magnocellularis: implications for phase locking in the avian auditory brainstem. J Neurosci 20:2954-2963.

Peña JL, Viete S, Albeck Y, Konishi M (1996) Tolerance to sound intensity of binaural coincidence detection in the nucleus laminaris of the owl. J Neurosci 16:7046-7054.

Rubel EW, Parks TN (1975) Organization and development of brain stem auditory nuclei of the chicken: tonotopic organization of $n$. magnocellularis and n. laminaris. J Comp Neurol 164:411-433.

Smith DJ, Rubel EW (1979) Organization and development of brain stem auditory nuclei of the chicken: dendritic gradients in nucleus laminaris. J Comp Neurol 186:213-239.

Smith PH, Joris PX, Carney LH, Yin TC (1991) Projections of physiologically characterized globular bushy cell axons from the cochlear nucleus of the cat. J Comp Neurol 304:387-407.

Smith PH, Joris PX, Yin TC (1998) Anatomy and physiology of principal cells of the medial nucleus of the trapezoid body (MNTB) of the cat. J Neurophysiol 79:3127-3142.

Sullivan WE, Konishi M (1984) Segregation of stimulus phase and intensity coding in the cochlear nucleus of the barn owl. J Neurosci 4:1787-1799.

Tollin DJ, Yin TC (2005) Interaural phase and level difference sensitivity in low-frequency neurons in the lateral superior olive. J Neurosci 25:10648-10657.

Warchol ME, Dallos P (1990) Neural coding in the chick cochlear nucleus. J Comp Physiol [A] 166:721-734.

Yang L, Monsivais P, Rubel EW (1999) The superior olivary nucleus and its influence on nucleus laminaris: a source of inhibitory feedback for coincidence detection in the avian auditory brainstem. J Neurosci 19:2313-2325.

Yin TC, Chan JC (1990) Interaural time sensitivity in medial superior olive of cat. J Neurophysiol 64:465-488.

Zhou Y, Carney LH, Colburn HS (2005) A model for interaural time difference sensitivity in the medial superior olive: interaction of excitatory and inhibitory synaptic inputs, channel dynamics, and cellular morphology. J Neurosci 25:3046-3058. 